\title{
INFINITELY MANY KNOTS WITH THE SAME POLYNOMIAL INVARIANT
}

\author{
TAIZO KANENOBU
}

\begin{abstract}
We give infinitely many examples of infinitely many knots in $S^{3}$ with the same recently discovered two-variable and Jones polynomials, but distinct Alexander module structures, which are hyperbolic, fibered, ribbon, of genus 2, and 3-bridge.
\end{abstract}

Two knots $K_{1}$ and $K_{2}$ in $S^{3}$ belong to the same isotopy type if there exists an orientation preserving homeomorphism of $S^{3}$ which maps $K_{1}$ onto $K_{2}$. We denote it by $K_{1} \approx K_{2}$. In 1984, V. Jones [9] discovered a very powerful polynomial invariant of the isotopy type of an oriented knot or link. Subsequently, the Jones polynomial was generalized to the two-variable polynomial invariant simultaneously and independently by Ocneanu [13], Lickorish and Millett [12], Hoste [8], and Freyd and Yetter. In this note we follow Lickorish and Millett. For a link $L$, the polynomial $L(l, m)$ is defined recursively by the following two conditions:

(I) If $L_{+}, L_{-}$and $L_{0}$ are three links with completely identical projections except at one crossing, where they are related as shown in Figure 1, then

$$
l L_{+}(l, m)+l^{-1} L_{-}(l, m)+m L_{0}(l, m)=0 .
$$

(II) If $K$ is a trivial knot, then $K(l, m)=1$.

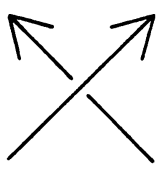

$L_{+}$

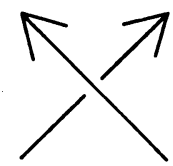

$L_{-}$

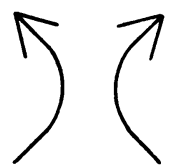

$L_{0}$

Let $\Delta_{L}(t), \nabla_{L}(z)$ and $V_{L}(t)$ be the Alexander polynomial, the Conway polynomial [4] and the Jones polynomial of a link $L$, respectively. They can be recovered from $L(l, m)$ by the formulas

where $i=\sqrt{-1}$.

$$
\begin{aligned}
& \Delta_{L}(t)=L\left(i, i\left(t^{1 / 2}-t^{-1 / 2}\right)\right), \\
& \nabla_{L}(t)=L(i, i z), \\
& V_{L}(t)=L\left(i t, i\left(t^{1 / 2}-t^{-1 / 2}\right)\right),
\end{aligned}
$$

Received by the editors January 4, 1985 and, in revised form, March 1, 1985.

198() Mathematics Subject Classification. Primary 57M25.

K'(l. words and phrases. Knot, two-variable polynomial invariant, Jones polynomial, Alexander module. 
Birman [2] gives examples of a pair of distinct closed 3-braids with the same Jones and Alexander polynomials. On the other hand, it is remarked in [12], as a consequence of (I) and (II), that these polynomials are invariants of the skein equivalence class [4], (cf. [7]) of the oriented link. For example, the KinoshitaTerasaka and the Conway 11-crossing knots with trivial Alexander polynomial have the same polynomials. Thus, using the pretzel knots [14], (cf. [1]), we have arbitrarily many distinct knots with the same polynomials.

In this note we prove

THEOREM. There exist infinitely many examples of infinitely many knots in $S^{3}$ with the same two-variable polynomial invariant and, therefore, the same Jones polynomial, but distinct Alexander module structures, which are hyperbolic, fibered, ribbon, of genus 2, and 3-bridge.

We consider the family of knots $K_{p, q}$ as shown in Figure 2, where the rectangle labeled $n$ stands for $|n|$ full-twists as shown in Figure 3.
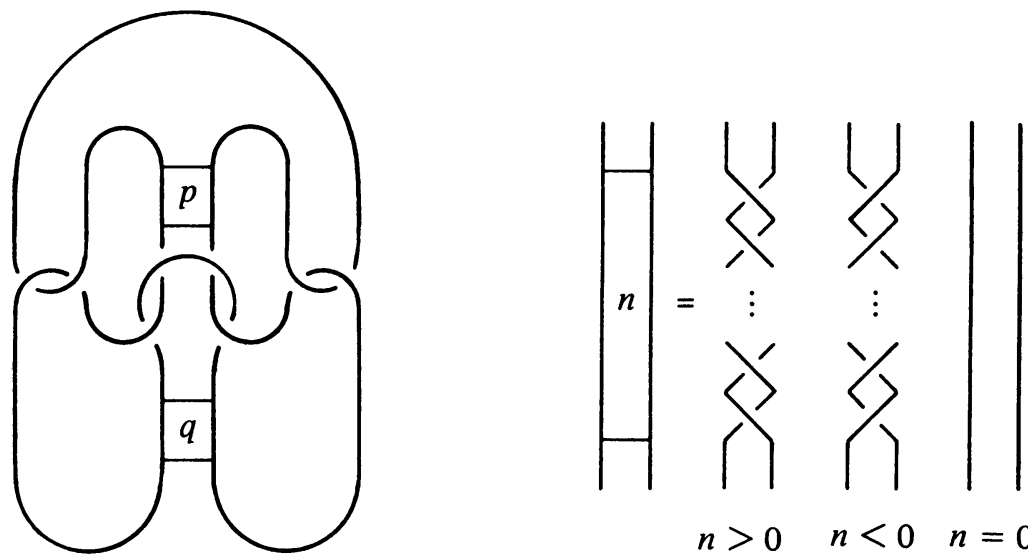

$$
n>0 \quad n<0 \quad n=0
$$

LEMMA $1 . K_{p, q} \approx K_{q, p}$.

Proof. It is easy to see that two knots of Figures 2 and 5 are isotopic to that of Figure 4. Turning over the projection (Figure 5), we obtain that of $K_{q, p}$.
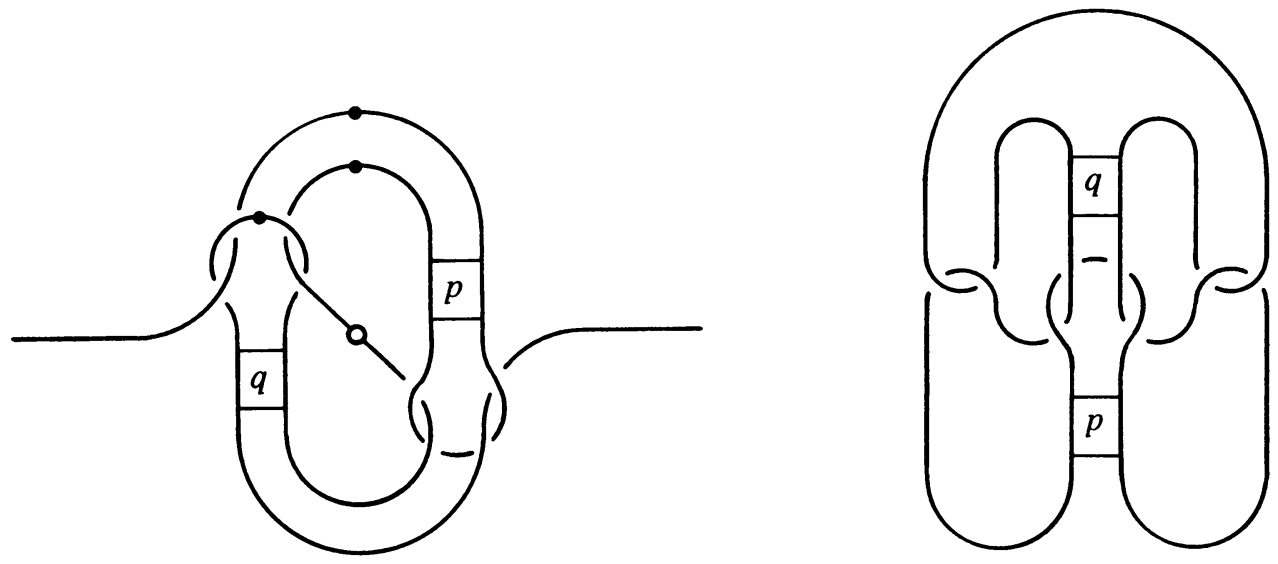
These knots were given by the author in [10], where $K_{p, p+1}(p=0,1,2, \ldots)$ were presented as the first specific example of infinitely many distinct fibered knots with the same Alexander module structure. The following are also mentioned in [10]: $K_{p . q}$ are fibered of genus 2, they are the symmetric unions of the figure-eight knot wound at two places [11], and, therefore, are ribbon knots. $K_{p, q}$ has the Alexander matrix

$$
\left[\begin{array}{cc}
t^{2}-3 t+1 & (p-q) t \\
0 & t^{2}-3 t+1
\end{array}\right]
$$

a presentation matrix for the Alexander module over the polynomial ring $\Lambda=$ $Z\left[t, t^{-1}\right]$. Here we show:

Lemma 2. $K_{p, q}$ and $K_{p^{\prime}, q^{\prime}}$ have the same Alexander module structure iff $|p-q|=$ $\left|p^{\prime}-q^{\prime}\right|$.

Proof. The second elementary ideal [6] is $\left(t^{2}-3 t+1, p-q\right)$. The $\Lambda$-module presentation $\Lambda /\left(t^{2}-3 t+1, p-q\right)$ has the infinite presentation as an abelian group:

$$
\left\langle\left\{a_{i}\right\} ;\left\{a_{i+2}-3 a_{i+1}+a_{i}=(p-q) a_{i}=0\right\}\right\rangle, \quad i=0,1,2, \ldots
$$

which is isomorphic to $Z_{|p-q|} \oplus Z_{|p-q|} ; Z_{0}$ means the infinite cyclic group. The result follows.

Now we calcuate the two-variable polynomial of $K_{p, q}$. By changing one of the crossings in the $p$ full-twists of Figure 2, we have

$$
l K_{p, q}(l, m)+l^{-1} K_{p-1, q}(l, m)+m \mu=0,
$$

where $\mu=-\left(l+l^{-1}\right) m^{-1}$ is the polynomial of the two-component trivial link. Then

$$
\begin{aligned}
K_{p, q}(l, m)-1 & =\left(-l^{-2}\right)\left(K_{p-1, q}(l, m)-1\right)=\left(-l^{-2}\right)^{p}\left(K_{0, q}(l, m)-1\right) \\
& =\left(-l^{-2}\right)^{p}\left(K_{q, 0}(l, m)-1\right)=\left(-l^{-2}\right)^{p+q}\left(K_{0,0}(l, m)-1\right) .
\end{aligned}
$$

$K_{0.0}$ is the product of two figure-eight knots, and so

$$
K_{0,0}(l, m)=\left(m^{2}-l^{2}-l^{-2}-1\right)^{2} .
$$

Thus we have

$$
K_{p, q}(l, m)=\left(-l^{-2}\right)^{p+q}\left(\left(m^{2}-l^{2}-l^{-2}-1\right)^{2}-1\right)+1,
$$

and so $K_{p, q}$ has Jones polynomial

$$
\left(t^{-2}\right)^{p+q}\left(\left(t^{2}-t+1-t^{-1}+t^{-2}\right)^{2}-1\right)+1 .
$$

Hence we obtain

LEMMA 3. $K_{p, q}$ and $K_{p^{\prime}, q^{\prime}}$ have the same two-variable and Jones polynomials iff $p+q=p^{\prime}+q^{\prime}$.

Combining Lemmas 1-3, we can completely classify the family of knots $K_{p, q}$. 
PROPOSITION. $K_{p, q} \approx K_{p^{\prime}, q^{\prime}}$ iff $(p, q)=\left(p^{\prime}, q^{\prime}\right)$ or $\left(q^{\prime}, p^{\prime}\right)$.

A knot $K$ is amphicheiral if $K \approx r K$, where $r K$ is the mirror image of $K$. In Figure 4, a half-rotation about a normal to the plane of projection at the origin $o$ takes $K_{p, q}$ to $r K_{-q,-p}$, see [18]. Thus we have

COROLlaRY. $K_{p, q}$ is amphicheiral iff $p+q=0$.

LEMMA 4. $K_{p, q}$ is a 3-bridge knot.

Proof. Figure 4 shows that $K_{p, q}$ has crookedness at most 3 (see [17, p. 115]), and so $K_{p, q}$ has bridge index at most 3 . We can readily compute to obtain [17] that the only 2-bridge knot of genus 2 with $\Delta(-1)= \pm 25$ is $(25,9)$ in Schubert's notation, or $8_{8}$ in the notation of Alexander and Briggs. Observing the Alexander polynomial, $K_{p, q}$ is not $8_{8}$. This completes the proof.

LEMMA 5. $K_{p, q}$ is hyperbolic iff $(p, q) \neq(0,0)$.

Proof. Riley [15] observes that a 3-bridge knot is either hyperbolic, a torus knot, or a product knot. It is easy to see that the only torus knot of genus 2 is that of type $(5,2)$, and so $K_{p, q}$ is not a torus knot. It is known $[3,5]$ that the only fibered knots of genus 1 are the trefoil and figure-eight knots. If $K_{p, q}$ is not prime, then $K_{p, q}$ is the product of two figure-eight knots. Thus $K_{0,0}$ is the only product knot in this family. This completes the proof.

Now the theorem is an immediate consequence of the lemmas.

REMARK. The invertibility of $K_{p, q}$ is unknown.

ACKNOWLEDGEMENT. I would like to thank Y. Nakanishi for showing me the proof of Lemma 2.

\section{REFERENCES}

1. R. E. Bedient, Double branched covers and pretzel knots, Pacific J. Math. 112 (1984), 265-272

2. J. S. Birman, On the Jones polynomial of closed 3-braids, Invent. Math. 81 (1985), 287-294.

3. (j. Burde and H. Zieschang, Neuwirthsche Knoten und Flächeabbildungen, Abh. Math. Sem. Univ. Hamburg 31 (1967), 239-246.

4. J. H. Conway, An enumeration of knots and links, and some of their algebraic properties. Computational Problems in Abstract Algebra, Pergamon Press, Oxford and New York, 1969, pp. 329-358.

5. F. González-Acuña, Dehn's construction on knots, Bol. Soc. Mat. Mexicana (2) 15 (1970), 58-77.

6. R. H. Crowell and R. H. Fox, Introduction to knot theory, Grad. Texts in Math., vol. 57. Springer-Verlag, New York and Berlin, 1977.

7. C. A. Giller, A family of links and the Conway calculus, Trans. Amer. Math. Soc. 270 (1982), $75-109$.

8. J. Hoste, A polynomial invariant of knots and links (preprint).

9. V. F. R. Jones, A polynomial invariant for knots via von Neumann algebra. Bull. Amer. Math. Soc. (N.S.) 12 (1985), 103-111.

10. T. Kanenobu, Module d'Alexander des noeuds fibrés et polynôme de Hosokaw'a des lacements fibrés, Math. Sem. Notes Kobe Univ. 9 (1981), 75-84.

11. S. Kinoshita and H. Terasaka, On unions of knots, Osaka Math. J. 9 (1957), 131-153.

12. W. B. R. Lickorish and K. C. Millettt, Topological invariants of knots and links (preprint).

13. A. Ocneanu, A polynomial invariant for knots: $A$ combinatorial and an algebraic approach. (preprint). 
14. R. L. Parris, Pretzel knots, Ph. D. Thesis, Princeton University, 1978.

15. R. Riley, An elliptical path from parabolic representation to hyperbolic structures, Lecture Notes in Math., vol. 722, Springer-Verlag, Berlin and New York, 1979, pp. 99-133.

16. D. Rolfsen, Knots and links, Math. Lecture Ser. 7, Publish or Perish, Berkeley, Calif., 1976.

17. L. Siebenmann, Exercises sur les noeuds rationnels, Orsay, 1975.

18. J. M. Van Buskirk, A class of negative-amphicheiral knots and their Alexander polynomials, Rocky Mountain J. Math. 13 (1983), 413-422.

Department of Mathematics, Kyushu University 33, Fukuoka, 812, Japan 